\title{
Ocorrência da osteocondrite dissecante na articulação tibiotársica em equinos da raça Brasileiro de Hipismo por meio da radiografia digital*
}

\section{Occurrence of osteochondritis dissecans in tibiotarsal joints of equines "Brasileiro de Hipismo" using digital radiography}

\author{
Marco Aurélio Gallo, ${ }^{* *}$ Luiz Fernando Rapp de Oliveira Pimentel, ${ }^{* *}$ André Luis do Valle De Zoppa ${ }^{* *}$
}

\begin{abstract}
Resumo
A osteocondrite dissecante (OCD) é uma doença ortopédica do desenvolvimento que acomete os humanos e os animais e é considerada como o distúrbio de desenvolvimento esquelético mais importante nos potros em crescimento. É uma falha no processo de ossificação endocondral, que compromete as cartilagens articular e de crescimento, principalmente a articulação tibiotársica. Sua ocorrência é importante para a equinocultura mundial pelo risco de produzir incapacitação nos cavalos atletas e de comprometer o bem-estar dos mesmos. O estudo radiográfico das articulações se mantém como método auxiliar de diagnóstico mais utilizado no campo e atualmente os equipamentos portáteis digitais permitem a rápida aquisição de imagens de alta qualidade. Os animais deste estudo foram escolhidos seguindo critérios descritos a seguir. A idade de três anos, a partir da qual as lesões se apresentam bem definidas e com remota possibilidade de reabsorção dos fragmentos. A sanidade, porque os animais sem sinais clínicos de OCD podem ter a avaliação radiográfica negligenciada e ser portadores sãos. A docilidade dos animais foi um critério de escolha, que permitiu um exame rápido e seguro, tanto para os operadores, quanto para os equipamentos utilizados. Pelo registro dos animais radiografados na Associação Brasileira dos Criadores de Cavalos de Hipismo (ABCCH) foi possível investigar a genealogia dos garanhões. O exame radiográfico consistiu na captação de duas projeções oblíquas bilaterais das articulações tibiotársicas. As imagens radiográficas digitais obtidas foram armazenadas e avaliadas posteriormente por profissional com experiência em radiologia e os achados classificaram os animais em positivos e negativos para OCD. Os resultados demonstraram ocorrência de 7,7\% (2/26) de OCD na articulação tibiotársica dos garanhões selecionados para o estudo, criados no estado de São Paulo.
\end{abstract}

Palavras-chave: cavalo, doença ortopédica, osteocondrose.

\begin{abstract}
Osteochondritis dissecans (OCD) is a developmental orthopaedic disease which can involves both humans and animals and is considered the most important skeletal disorder in growing foals. It is a failure of the endochondral ossification which affects both articular and growth-plate cartilages, primary the tibiotarsal joint. Its occurrence is remarkable for the worldwide horse industry for the risk in disabling sport horses and concerns animal welfare. The radiographic investigation of joints remains the most common diagnostic auxiliary method employed in the field and updated portable digital X-rays machines provide fast acquisition of high quality images. The animals in this study were chosen by the following criteria. The age of three years old when the articular fragments are well defined and stable with low possibilities of resorption. The soundness because horses with no clinical signs are normally neglected and misdiagnosed for OCD. The good-behaviour of the horses provided a safe radiographic approach either for the operators or for the equipment. The genealogical background of the animals was investigated by their breeding record in the Brazilian Association of Sport Horses (ABCCH). The radiographic evaluation consisted in two bilateral oblique views of both tibiotarsal joints. The digital images were stored and further interpreted by an expert radiologist and the results scored the animals into a positive and a negative group for OCD. The results yielded an incidence of $7.7 \%(2 / 26)$ of tibiotarsal OCD in the stallions selected for this study, all of them bred in São Paulo State.
\end{abstract}

Keywords: horses, orthopaedic disease, osteochondrosis.

\footnotetext{
${ }^{*}$ Recebido em 24 de julho de 2013 e aceito em 6 de janeiro de 2014.

**Faculdade de Medicina Veterinária e Zootecnia da Universidade de São Paulo.

E-mail para contato: maureliogallo@gmail.com.
} 


\section{Introdução}

A osteocondrite dissecante (OCD), defeito na ossificação endocondral, é mundialmente reconhecida como uma das mais importantes alterações ortopédicas que acometem os mamíferos em crescimento (Al-Hizab et al., 2002; Van Weeren, 2006). Acomete os equinos, tornando-a espécie de escolha como modelo de estudo para tal afecção (Van Weeren, 2006). $\mathrm{O}$ aparecimento da OCD em plantéis de determinadas raças de equinos pode levar a perdas econômicas, pois pode resultar em custo elevado no tratamento e controle ou até mesmo no descarte do animal, na dependência de sua função.

Os equinos com OCD podem apresentar efusão sinovial, claudicação leve (Harrison; Edwards, 1996; Brink et al., 2010), diminuição do rendimento atlético e progredir para osteoartrose da articulação acometida nos casos persistentes (Van Weeren, 2006; Olstad et al., 2007).

A articulação tibiotársica nos equinos, por ser a mais acometida por OCD, possui maior importância na clínica do aparelho locomotor (McllWraight, 1987). Em alguns animais ela pode apresentar-se de forma assintomática, dificultando o diagnóstico precoce (Brink et al., 2010). Além disso, a dor persistente nos animais de competição sintomáticos torna-se motivo de preocupação para os médicos veterinários, no compromisso de promover o bem-estar animal (Al-Hizab et al., 2002). As raças de origem europeia destinadas ao hipismo (Warmbloods) são as mais predispostas (Van Weeren, 2006; Stock e Distl, 2007).

A OCD é o resultado de falha na ossificação endocondral, acometendo o complexo articular-epifisário (McllRaight, 1987; Jeffcott, 1991). Alguns estudos citam ausência de canais cartilaginosos em equinos entre 6 e 7 meses de idade, sendo que, a partir dessa idade, os condrócitos seriam nutridos exclusivamente pelo líquido sinovial (Carlson et al. 1995; Shingleton et al., 1997). A falha causada durante o processo de ossificação endocondral pode resultar em reparação espontânea ou progredir em OCD, quando aplicado o componente biomecânico (Watikins, 1999).

Estudos radiográficos mensais em potros bem jovens demonstraram OCD nas articulações tibiotársicas e femorotibiopatelares, em linhagens holandesas, podendo algumas delas regredir ou permanecer inalteradas por volta do quinto mês de vida, sendo esta considerada idade limite (Dick et al. 1999).

A etiopatogenia da OCD não está totalmente esclarecida; por isso, inúmeros trabalhos demonstram sua origem multifatorial (Jeffcott, 1991; Carlson et al. 1995; Al-Hizab et al., 2002). Fatores genéticos (Van Weeren, 2006; Wittwer et al., 2006; Dierks et al., 2007; Wittwer et al. 2009), biomecânicos (Mcllwraight, 1987; Jeffcott, 1991; Van Weeren, 2006; Ytrehus et al. 2007), enzimáticos por ação de metalanoproteinases (Al-Hizab et al., 2002) e finalmente nutricionais, como a resposta glicêmica (Pagan, 2005) e desbalanceamento de microelementos estão envolvidos no desenvolvimento da doença (Van Weeren, 2006). Esta pode ainda ser de caráter congênito, pois outros estudos revelaram alterações histológicas e moleculares cartilagíneas precoces em fetos e neonatos (Lecocq et al., 2008).

O estudo radiográfico ainda é o meio auxiliar de eleição para o diagnóstico da OCD tibiotársica. Nesta articulação os principais sítios anatômicos afetados são a crista dorsal intermédia da tíbia e a porção distal da crista troclear lateral do osso tarsotibial (talus)
(Shelley e Dyson, 1984; Buttler, et al., 1993). Segundo Shelley e Dyson (1984), o estudo radiográfico da região tibiotársica deve ser realizado por 4 projeções distintas: a dorsoplantar (DPI), a lateromedial (LM), dorsolateral-plantaromedial oblíqua (DL-PIMO) e dorsomedial-plantarolateral oblíqua(DM-PILO). No entanto, um estudo feito na Itália, com 350 equinos da raça Maremmano, na idade de 3 anos, demonstrou que apenas 2 projeções radiográficas oblíquas são suficientes para a triagem da OCD na articulação tibiotársica (Pieramati et al., 2003).

\section{Material e métodos}

Este trabalho obteve aceitação e protocolo $n^{\circ} 2025 / 2010$ pela "Comissão de Ética no uso de animais" da Faculdade de Medicina Veterinária e Zootecnia da Universidade de São Paulo. Por meio de anamnese e de inspeção visual, foram selecionados 26 equinos dentro de plantéis de criadores localizados num raio de até 100 quilômetros da cidade de São Paulo. Os animais foram radiografados nas propriedades de origem entre julho de 2008 e outubro de 2010. Os equinos selecionados eram machos inteiros, com idade entre 27 e 46 meses, todos hígidos, com os dois testículos na bolsa escrotal, apresentando bom escore corpóreo. Todos foram registrados na $\mathrm{ABCCH}$ (Associação Brasileira de Criadores de Cavalos de Hipismo) e produtos de inseminação artificial com sêmen de origem europeia, considerados "OCD-free" (isentos da transmissão da OCD) pelas centrais onde foram adquiridos. Para a aquisição das imagens radiográficas foram utilizados equipamentos portáteis, a saber: um emissor de raios $X$ portátil de alta frequência ORANGE ${ }^{\circledR}$, modelo $10040 \mathrm{HF}$, juntamente com um processador de imagens radiográficas digitais diretas com painel plano de selênio amorfo, conectado por cabo, da marca EKLIN $^{\circledR}$, modelo Mark III. Os animais foram submetidos ao exame radiográfico em estação, naturalmente posicionados com os quatro membros em apoio pleno, por meio de duas projeções oblíquas bilateralmente, DL-PIMO e DM-PILO respectivamente, das articulações tibiotársicas conforme descrito por Pieramati e colaboradores (2003). Os fatores de exposição padronizados para todos os animais foram $92 \mathrm{kVp}$ e 2,2 mAs, em ambas as projeções oblíquas. Todas as imagens em padrão DICOM (Digital Imaging Communications in Medicine) foram gravadas no processador digital e posteriormente analisadas (Figura 1). O programa utilizado no processador foi o E-FILM ${ }^{\circledR}$. Após a análise criteriosa, os animais avaliados foram divididos em dois grupos distintos: positivos, para os que possuíam achados radiográficos típicos de OCD na articulação tibiotársica, que são fragmentos osteocondrais liberados da crista intermédia dorsal da tíbia e/ou fragmentos osteocondrais liberados das cristas trocleares do talus; e negativos, para os que não possuíam achados radiográficos de OCD. Demais possíveis fragmentos intra-articulares ou não, que não os supramencionados seriam considerados como negativos para OCD. A sedação não foi utilizada nos potros, pelo fato de os mesmos terem cooperado com o estudo, aliado ao fato de ter sido empregada uma técnica radiográfica com tempo de exposição extremamente rápido para a aquisição das imagens.

\section{Resultados}

A média de idade dos equinos selecionados foi de 36,3 meses. Quanto ao país origem do sêmen importado obteve-se a seguinte distribuição: $65,38 \%$ de sêmen holandês, $19,24 \%$ de 
sêmen alemão e $15,38 \%$ de sêmen francês. Dos 26 animais radiografados, dois foram considerados positivos $(7,7 \%)$ para os achados radiográficos de OCD na articulação tibiotársica. Um deles apresentou fragmento osteocondral próximo ao sítio anatômico de origem, de formato elipsoidal, destacado da crista dorsal intermédia da cóclea tibial direita, que é um local anatômico típico da OCD (Figura 1). O outro animal positivo apresentou fragmento osteocondral solto no espaço articular, na altura da articulação intertarsiana proximal esquerda, dorsalmente as tróclea do osso tarsotibial (talus). A projeção radiográfica que melhor colaborou com a visualização dos achados de OCD nos 2 casos foi a DM-PILO. Dos animais avaliados neste estudo, três foram aprovados como garanhões pela $\mathrm{ABCCH}$ e negativos para OCD pelo critério proposto neste trabalho.

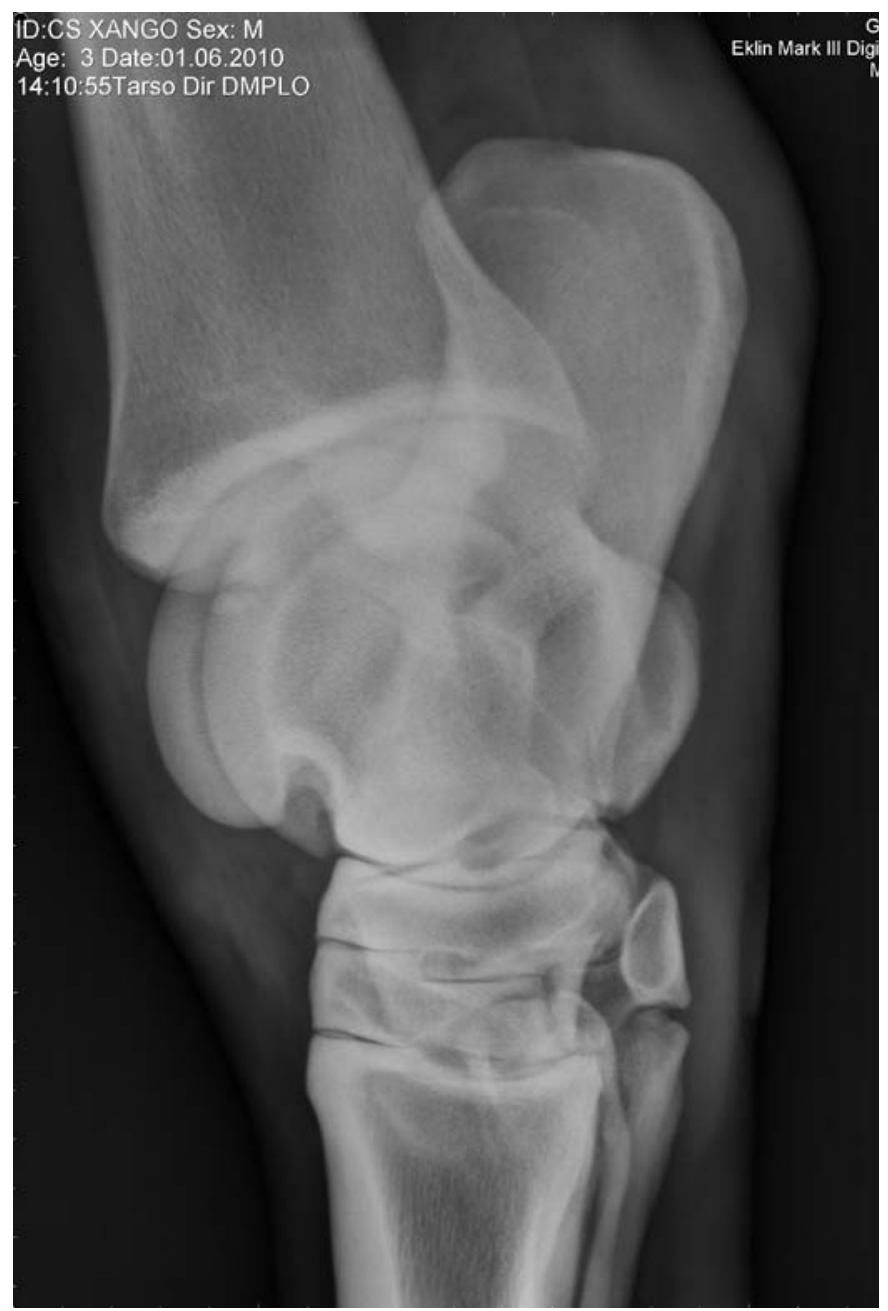

Figura 1: Equino da raça Brasileiro de Hipismo de 42 meses de idade. Projeção radiográfica DM-PILO do tarso direito. A seta indica alteração típica da OCD equina: separação de fragmento osteocondral da crista dorsal intermédia da tíbia e erosão subcondral

\section{Discussão}

A raça Brasileiro de Hipismo, fundada em 1977, foi escolhida por estar em formação e ser oriunda de plantéis europeus (raças formadoras), cuja ocorrência da OCD é controlada. Como raças formadoras da $\mathrm{BH}$, as mais utilizadas foram Puro-Sangue Inglês, Hanoveriano, Westfalen, Holsteiner, Trakehner, Oldenburg,
Orloff, Rheinland, Anglo-Árabe, Sela Belga, Sela Francês, Sela Holandês, Sela Argentino e Zangersheide (Dias, et al., 2000). As associações de criadores de equinos no exterior, como a Associação Alemã do Cavalo Hanoveriano e a Associação Nacional do Cavalo de Sela Holandês (KWPN) promovem o controle da OCD em várias articulações, por meio de exame radiográfico dos reprodutores em potencial, etapa importante no julgamento para a aprovação dos reprodutores. No Brasil, a $\mathrm{ABCCH}$ exige apresentação de exame radiográfico dos ossos naviculares dos membros torácicos e das articulações tarsais dos candidatos para aprovação de garanhões. A presença de OCD nos animais submetidos à seleção de garanhões não é desclassificatória. Este critério nada rigoroso pode permitir a aprovação de garanhões OCD positivos, o que possibilita a transmissão e perpetuação da afecção a futuras gerações. Antes de se exigir o controle radiográfico aos candidatos a aprovação de garanhões pela $\mathrm{ABCCH}$, houve a possibilidade de animais assintomáticos terem sido aprovados com OCD. Além da adoção de critérios mais rigorosos na avaliação radiográfica do candidato a garanhão, também deveria ser exigido exame radiográfico de seus descendentes. Este critério poderia melhor testar garanhões aprovados, que não apresentaram achados radiográficos da OCD por terem sido submetidos à retirada cirúrgica de fragmentos antes do julgamento. Foram realizadas duas projeções radiográficas neste estudo, as oblíquas DL-PIMO e a DM-PILO, assim como estudo realizado na Itália com equinos da raça Maremmano (Pieramati et al., 2003). Reduzindo-se o número de projeções e consequentemente dos custos, o valor poupado poderia estimular uma abordagem radiográfica mais ampla, incluindo-se outras articulações a serem investigadas em um mesmo animal, como a articulação femorotibiopatelar.

Outro aspecto a ser considerado é o fato de ser muito precoce nos equinos o aparecimento de alterações da OCD (Dick et al., 1999; Olstad et al., 2007; Lecocq et al., 2008). Isso sugere a criação de um modelo de triagem que poderia ser estimulado aos criadores brasileiros, com aquisições de imagens sequenciais nos potros para melhor entendimento da evolução da OCD no Brasil, associado a outros métodos de detecção da doença, sendo um estudo multidisciplinar mais sensível e com maior grau de êxito na detecção e controle da OCD.

Os valores achados neste estudo foram abaixo dos valores encontrados nos trabalhos de autores europeus (Pieramati et al., 2003; Wittwer et al., 2006; Stock, Distl 2007). Todavia, o objetivo foi investigar uma população de equinos assintomáticos. Essa escolha pode ter excluído do estudo muitos animais positivos para a OCD.

O diagnóstico da OCD, pela simples presença de fragmento osteocondral intra-articular aleatoriamente, sem critérios extremamente fidedignos, pode induzir a erros de interpretação. Um erro de diagnóstico e consequentemente nas estatísticas da ocorrência da OCD seria caracterizar, como tal afecção, a simples presença de fraturas osteocondrais induzidas pelo exercício, desconhecendo-se os achados patognomônicos.

A classificação de sêmen "OCD-free" não exclui totalmente a possibilidade de transmissão da OCD por via genética, já que as éguas não são ainda controladas, responsáveis por $50 \%$ da transmissão genética aos seus descendentes e tampouco exclui os fatores ambientais de serem determinantes para o aparecimento da OCD, por ser de caráter multifatorial e, até agora, com fatores predisponentes e determinantes ainda obscuros. 


\section{Conclusão}

Conclui-se que há necessidade de mais estudos sobre a OCD na articulação tibiotársica dos equinos no Brasil, para ser melhor entendida quanto a sua ocorrência e etiologia. Recomenda-se a inclusão de outros locais, de outras raças formadoras, com ambos os sexos e com maior amostragem, para uma análise mais fidedigna sobre a ocorrência desta afecção no território nacional.

\section{Referências}

AL-HIZAB, F.; CLEGG, P. D.; THOMPSON C. C.; CARTER, S. D. Microscopic localization of active gelatinases in equine osteochondritis dissecans (OCD) cartilage. Osteoarthritis and Cartilage, v. 10, n. 8, p. 653-661, 2002.

BRINK, P.; SKYDSGAARD, M.; TEIGE, J.; TVERDAL, A.; DOLVIK, N. I. Association between clinical signs and histopathologic changes in the synovium of the tarsocrural joint of horses with osteochondritis dissecans of the tibia. American Journal of Veterinary Research, v. 71, n. 1, p. 47-54, 2010.

BUTLER, J. A.; COLLES, C. M.; DYSON, S. J.; KOLD, S. E.; POULOS, P. W. The tarsus. In: Clinical radiology of the horse. Oxford: Blackwell Science, 1993. chap. 5, p. 211-245.

CARLSON, C. S.; HILLEY, H. D.; MEUTEN, D. J. Osteochondrosis of the articular-epiphyseal cartilage complex in young horses: evidence for a defect in cartilage canal blood supply. Veterinary Pathology, v. 32, n. 6, p. 641-647, 1995.

DIAS, I. M. G.; BERGMANN, J. A. G.; REZENDE, A. C. C.; CASTRO, G. A. F. Formação e Estrutura Populacional do Equino Brasileiro de Hipismo. Arquivo Brasileiro de Medicina Veterinária e Zootecnia, v. 52, n. 6, 2000. Disponível em: <http://www.scielo.br/ scielo.php?pid=S0102-09352000000600016\&script=sci_arttext $>$. Acesso em: 30 mai. 2010.

DICK, K. J.; ENZERINK, E.; VAN WEEREN, P. R. Radiographic development of osteochondral abnormalities, in the hock and stifle of Dutch Warmblood foals, from age 1 to 11 months. Equine Veterinary Journal, v. 31, n. 531, p. 9-15, 1999. Supplement.

DIERKS, C.; LÖHRING, K.; LAMPE, V.; WITTWER, C.; DRÖGRMÜLLER, C.; DISTL, O. Genome-wide search for markers associated with osteochondrosis in Hanoverian Warmblood horses. Mamm Genome, v. 18, p. 739-747, 2007.

GETTY, R. Osteologia. In: Sisson/Grossman anatomia dos animais domésticos. 5 ed. Rio de Janeiro: Guanabara Koogan, 1986. v. 1 , cap. 15 , p. $283-292$.

HARRISON, L. J.; EDWARDS, G. B. Radiographic investigation of osteochondrosis. Equine Veterinary Education, v. 8, n. 3, p. 172176, 1996.

JEFFCOTT, L. B. Osteochondrosis in the horse - searching for the key to pathogenesis. Equine Veterinary Journal, v. 23, n. 5, p. 331 338, 1991.

LECOCQ, M.; GIRARD, C. A.; FOGARTY, U.; BEAUCHAMP, G.; RICHARD, H.; LAVERTY, S. Cartilage matrix changes in the developing epiphysis: early events on the pathway to equine osteochondrosis? Equine Veterinary Journal, v. 40, n. 5, p. $442-$ 454, 2008.
A triagem da OCD tibiotársica por meio de radiografia digital com apenas duas projeções oblíquas mostrou-se um modelo rápido e pouco oneroso. Sua utilização poderia ser estimulada para os criadores no campo, contribuindo para o controle da doença pela obtenção de mais informação sobre sua ocorrência e melhoria do plantel nacional.

MCILWRAIGHT, C. W. Diseases of joints, tendons, ligaments and related structures. In: STASHAK, T. Adams' lameness in horses. $4^{\text {th }}$ ed. Philadelphia: Lea \& Febiger, 1987. chap. 7, p. 396-419.

OLSTAD, K.; YTREHUS, B.; EKMAN, S.; CARLSON, C. S.; DOLVIK, N. I. Early lesions of osteochondrosis in the distal tibia of foals. Journal of Orthopaedic Research, v. 25, n. 8, p. 1094-1105, 2007.

PAGAN, J. D. The relationship between glycemic response and the incidence of OCD in thoroughbred weanlings: a field study. In: PAGAN, J. D.; GEOR, R. J. Advances in equine nutrition III. Nottingham: Nottingham University Press, 2005. p. 433-437.

PIERAMATI, C.; PEPE, M.; SILVESTRELLI, M.; BOLLA, A. Heritability estimation of osteochondrosis dissecans in Maremmano horses. Livestock Production Science, v. 79, n. 2-3, p. 249-255, 2003.

SHELLEY, J.; DYSON, S. Interpreting radiographs 5. radiology of the equine hock. Equine Veterinary Journal, v. 16, p. 488-495, 1984.

SHINGLETON, W. D.; MACKIE, E. J.; CAWSTON, T. E.; JEFFCOTT, L. B. Cartilage canals in equine articular/epiphyseal growth cartilage and a possible association with dyschondroplasia. Equine Veterinary Journal, v. 29, n. 5, p. 360-364, 1997.

STOCK, K. F.; DISTL, O. Genetic correlations between performance traits and radiographic findings in the limbs of German Warmblood riding horses. Journal of Animal Science, v. 85, p. 31-41, 2007.

VAN WEEREN, P. R. van Etiology, diagnosis and treatment of OC(D). Clinical Techniques in Equine Practice, v. 5, p. 248-258, 2006.

WATKINS, J. P. Osteochondrosis, In: AEUR, J. Equine surgery. $2^{\text {nd }}$ ed. Philadelphia: W.B. Saunders, 1999. p. 765-778.

WITTWER, C.; HAMANN, H.; DISTL, O. The candidate gene $X I R P 2$ at a quantitative gene locus in equine chromossome 18 associated with osteochondrosis in fetlock and hock joints of South German Coldblood horses. Journal of Heredity, v. 100, n. 4, p. 481486, 2009.

WITTWER, C.; HAMANN, H.; ROSENBERGER, E.; DISTL, O. Prevalence of osteochondrosis in the limb joints of South German Coldblood horses. Journal of the Veterinary Medicine Association, v. 53 , n. 10 , p. 531-539, 2006.

YTREHUS, B.; CARLSON, C. S.; EKMAN, S. Etiology and pathogenesis of osteochondrosis. Veterinary Pathology, v. 44, n. 4, p. 429-448, 2007. 